\title{
The Self-Injected Laser for Picosecond Time-Resolved Spectroscopy
}

\author{
F. ARMANI, F. DE MARTINI and P. MATALONI \\ Quantum Optics Laboratory, Istituto di Fisica "G. Marconi" Università di Roma, \\ Roma 00185, Italy
}

The principles of operation and the characteristics of the self-injected picosecond laser are presented. We show that in spite of its simple design our device is able to generate very high power pulses in the picosecond domain. This warrants the use of this laser for time resolved spectroscopy in the picosecond domain.

\section{INTRODUCTION}

In this paper we present the principles of operation and the characteristics of the first self-injected picosecond laser. In particular, the laser will be presented in its latest version including a double active medium in the optical cavity. The combined effects of the selfinjection process, ${ }^{1,2}$ of the large pulse compression due to pulse propagation in a saturable absorber in the cavity ${ }^{3,4}$ and of the relative large amplification bandwidth of the active medium lead to the generation of $\sim 5 \mathrm{ps}, 1 \mathrm{GW}, \lambda \simeq 1.06 \mu$ optical pulse with $\sim 10 \%$ pulse to pulse energy stability at low threshold and good repetition rate. Our laser presents several advantages with respect to the usual modelocked lasers. These advantages are consequential of the new concepts on which the laser operation is based. In fact the good pulse stability, the large output pulse intensity, the high reproducibility and the simple and rather inexpensive design of our device are to be ascribed to the process of pulse formation itself which acts, originally, on a coherent pulse generated in the cavity by a normal Q-switching effect. Consequently, critical conditions related to the preparation in the 
cavity of phase-locked modes determining the complicated dynamics of the chaotic-coherent evolution of the pulse statistics about laser threshold, are absent in our system, together with the related output instabilities.

The optical design of our system is the same of a usual Pockels Cell (PC) Q-switched (Q-sw) laser. We can say that almost any commercially available, or home made, (PC) Q-sw laser can be transformed at low cost, by our technique, in a reliable, high power picosecond laser. In fact, no Brewster-angle cut components or careful optical design are needed and several active elements can be inserted in the cavity with no appreciable disturbance on the output. A little more sophisticated electronics for the Pockels Cell driving circuit is needed however as this device is requested to perform several functions with low switching time i.e., Q-switching, self-injection (or cavity-flipping), cavity dumping.

From a very qualitative view point the overall operation of the laser can be understood as follows. A normal Q-switching action is triggered by $\mathrm{PC}$ at time $t=t_{0}$ in a laser cavity provided with two high reflectivity optical end mirrors with $\bar{R} \sim 100 \%$ at $\lambda=1.06 \mu$. Owing to the high $\mathbf{Q}$ of the cavity, the laser threshold can be very low. At a successive time $t_{1}\left(t_{1}-t_{0} \equiv \tau=\right.$ few nanoseconds) a second fast electrical pulse $V(t)$ is applied to PC giving rise to the so called cavityflipping operation. ${ }^{1,2}$ This corresponds essentially to dumping out of the cavity a large time portion of the coherent (i.e., over threshold) Q-sw radiation pulse which is now growing rather slowly in the laser cavity (typically in $\sim 20 \mathrm{~ns}$ ).

At the end of this operation only a rather short ( $<1 \mathrm{~ns})$ coherent "seed" pulse is kept in the cavity, bouncing back and forth between the two $R \sim 100 \%$ end mirrors through the active medium. If $\left(t_{1}-t_{0}\right)$ is short enough to frustrate the normal evolution of the Q-sw pulse in the cavity just after the threshold condition has been reached at $t=t_{0}$ and if the fluorescence time $T_{1}$ associated with the relevant atomic transition in the active medium is larger than the pulse length obtained in a normal Q-sw operation, the population inversion stored in the active medium at time $t=t_{1}$ can be very close to the one at $t=t_{0}$ i.e., the one that would determine the total energy of the pulse in normal Q-switching operation. Since in our laser this population inversion is now available for amplification of a subnanosecond coherent seed pulse during its multiple passages in the active medium, we 
conclude that virtually the entire energy that would have been emitted in normal Q-switching operation in absence of cavity flipping will be finally compressed in a such shorter pulse. This gives rise, at the end of the amplification process, to the generation of a very high power pulse of subnanosecond duration. We note, at this stage, that the time duration of the output pulse can be easily selected by varying the position of (PC) in the cavity (Figure 1) and/or the duration of the electrical pulse applied to PC which corresponds to the cavity flipping process. ${ }^{2,5}$ In practice one can obtain an adiabatic pulse shortening from about $10-20 \mathrm{~ns}$ down to $\sim 0.5 \mathrm{~ns}$, this last figure being limited by the finite rise time of PC. ${ }^{5}$ Further pulse shortening down to the picosecond domain is obtained by inserting in the cavity a saturable dye flowing cell that provides a nonlinear compression effect. We found experimentally that, by this technique, we can generate high power optical pulses with duration limited by the amplification bandwidth of the active medium. Using a single Nd:Yag rod in our laser we were able to generate $15 \mathrm{ps}, \lambda=1.06 \mu$ pulses in the gigawatt range. ${ }^{4} \mathrm{We}$ note, in this connection, that our Nd:Yag picosecond laser had a very low threshold due to the high $Q$ of the optical cavity during the seed pulse amplification process and to the characteristics of the Yag medium. ${ }^{6}$ We could still keep these advantages of the Yag and, at the same time, rise the output peak power and further shorten the pulse by adding a second "amplifier" Nd:Glass rod in the cavity (Figure 1).

The flash-pumping level of this rod is kept at a level that doesn't allow the onset of laser action in absence of the unpumped Yag rod. On the other hand, the large stored energy ${ }^{6}$ and the much larger amplification bandwidth with respect to Yag (about a factor 40$)^{7}$ are

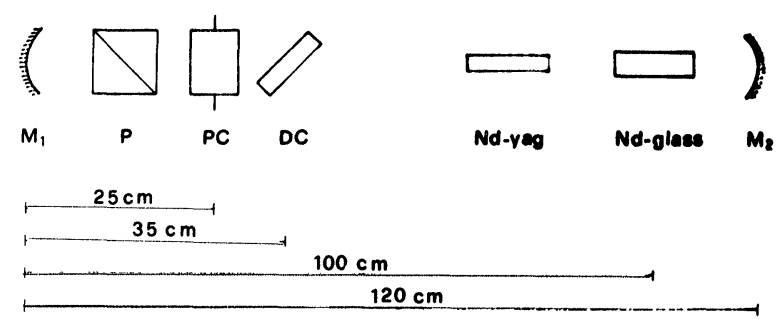

FIGURE 1 Laser cavity scheme used for regenerative pulse compression in the self injected laser. 
used to improve substantially the performances of our device. We have already noted that the principles of operation of our laser differ from the ones of Mode-locking. ${ }^{8}$ From an operational view point this result in a highly stable and reliable behavior of our device. Furthermore picosecond pulse operation of typically high threshold active media, like ruby, can be easily achieved by our method. It is well known that for this last material a good and useful mode-locking operation is very difficult to achieve. ${ }^{9}$

\section{EXPERIMENTAL}

In our laser two $A R$ coated rods $(3 \times 50 \mathrm{~mm} \mathrm{Nd:Yag} \mathrm{and} 4.5 \times$ $60 \mathrm{~mm} \mathrm{Nd}$ :phosphate glass ${ }^{7}$ ) were pumped independently in double ellipitical cavities by two couples of simmered flashlamps. For our present application the optical cavity was designed in such a way as to provide the adequate balance between the laser intensity seen by the active media and by the saturable dye flowing in a cell. This is required by the different values of the gain and absorption cross sections for the two media, respectively, and by the need of reaching simultaneously a regime of saturation for both gain and absorption in order to obtain a good compression efficiency. ${ }^{3,4}$ Two equally totally reflecting spherical mirrors with radii $R_{1}=99.9 \mathrm{~cm}$ and $R_{2}=67.5 \mathrm{~cm}$ determined the geometrical size of the cavity. With the geometrical parameters given in Figure 1, the ratio of beam areas in the dye cell and in the rods was $r=7,6$. This includes the effect of the Brewster angle tilting of the dye cell (DC) (thickness $=1 \mathrm{~mm}$ ). Due to rapid laser induced degradation of the dye it was found necessary to flow it through the cell. This one has been designed in such a way as to minimize turbulence effects in order to obtain good shot to shot reproducibility. The dye we used was the Eastman-Kodak No. 9740 Q-switching solution in dichloroetane. The dye concentration was adjusted to an adequate value different from the one which caused self Q-switching and mode-locking in the laser. The dye concentration corresponded to a small signal transmission in the cell: $T=10^{-(0.55)}$ The Pockels Cell (PC) was a Lasermetrics 1057 FV driven by a krypton circuit capable of delivering the wave form which is necessary for a combined Q-switching, cavity flipping and cavity dumping operations. The corresponding sequence of three electrical pulses that are applied to PC is shown in Figure $2 a$ while in Figure $2 b$ and $2 c$ the 

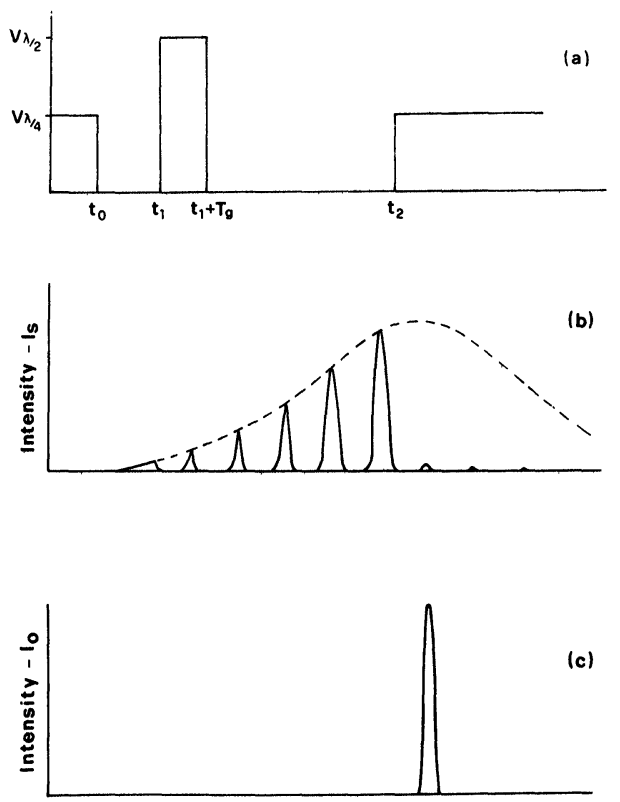

FIGURE 2 Time diagrams for self injection and cavity dumping: (a) Voltage applied to Pockels Cell; (b) pulse build up inside the cavity; (c) single output dumped pulse.

building up of the optical pulse in the cavity and the effect of cavity dumping are shown schematically. We have verified that, in subnanosecond operation of the laser (i.e., with no dye cell inserted in the cavity), the seed pulse envelope of Figure $2 b$ ) reproduces the Q-switched pulse that one can obtain by driving PC by just the first pulse of sequence $2 \mathrm{a}$ ).

When the laser was operating in the picosecond regime, the optimization of the pulse compression required a somewhat careful adjustment of the pumping energy delivered to the rods in correspondence with the dye concentration. In that case the shape of the optical pulse envelope in the cavity was determined by the nonlinear processes of pulse compression and amplification which are at the basis of the behavior of our device. These effects are shown in the oscilloscope traces shown in Figure 3. A fast ITL 1850 vacuum photodiode and a Tektronix $519 \mathrm{TW}$ oscilloscope were used. The overall risetime of the detection system was 320 ps. When the laser was operating in the 


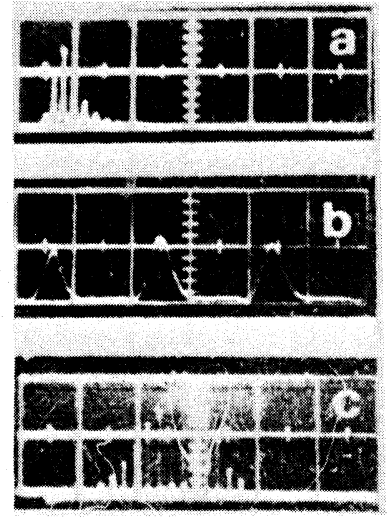

FIGURE 3 (a) Pulse train in self injection operation without dye in the cavity $(50 \mathrm{~ns} / \mathrm{div})$. (b) Same conditions as in $a$ ) but showing the self injected pulse duration $(5 \mathrm{~ns} / \mathrm{div})$. (c) Pulse train in self injection operation with dye solution in the cavity $(50 \mathrm{~ns} / \mathrm{div})$.

picosecond regime, the shape of the optical pulse envelope in the cavity gave a fair indication of the good pulse shortening performance of the laser. The slow rise of the leading edge of the envelope at low pulse power indicates that the overall, low level, laser gain is small. This is a condition for reaching the maximum intensity of the train with a large number of shortening passages. In our case the pulse made about 100 passages since the peak of the envelope of Figure $3 \mathrm{c}$ is delayed with respect to the self injection operation by $\sim 500 \mathrm{~ns}$. In addition to that, in this regime, each passages of the pulse in the dye is more effective for compression since the pulse intensity is maintained in the range where the dye nonlinearity is stronger. It is very important not to saturate completely the dye in the first passages since the completely saturated dye has no pulse compression capability. After the pulse reaches its maximum intensity it falls down quite fastly since, as the power decreases, the dye gets less saturated and the cavity loss increases correspondingly.

The timing of the self injection operation was found to be very important for obtaining good pulse compression. If self injection is triggered too early, the initial seed pulse is not intense enough to start the process of dye saturation. On the other hand, if the self injection is triggered too late the seed pulse makes too small a number of compressing passages through the dye. 
A substantial enhancement of the pulse compression effect has been obtained by taking advantage of the relatively large amplification bandwidth of the Nd:phosphate glass. We could verify, by varying the flashpumping condition of the glass rod, that a decrease of a factor $\sim 3$ of output pulse width (down to $\sim 5 \mathrm{ps}$ ) could be reached in our system by operating the Yag and glass media in optimum conditions. The measurement of the picosecond pulse length has been carried out independently by the two methods of two-photon fluorescence and background free autocorrelation by SHG. ${ }^{10}$ The result of a standard triangular two photon fluorescence measurement on our device has already been reported by us. ${ }^{4}$ In Figure 4 the II order,

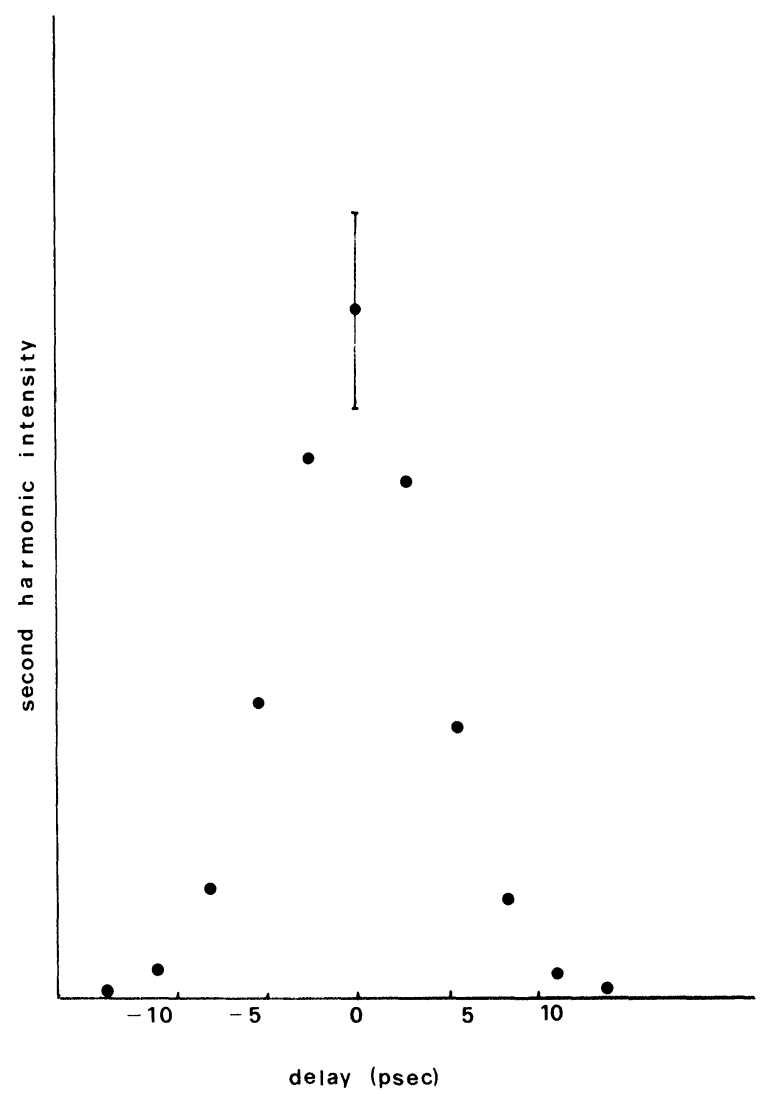

FIGURE 4 SH background-free autocorrelation curve. 
background free, autocorrelation curve, obtained in $1 \mathrm{~mm}$ KDP crystal, is reported. A duration of $5 \mathrm{ps}$ is obtained by assuming a gaussian shape of the output pulse.

\section{CONCLUSIONS}

In summary we have presented a new technique for production of high power picosecond pulses. In the present application we observed a pulse compression by a factor $\sim 200$ with respect to the normal self injection operation, while the peak power gain in the compression process was $\sim 70$. This implied an energy reduction in this process by a factor of only $\sim 3$. The obtained short pulses were very stable and showed low jitter $( \pm 10 \mathrm{~ns})$ relative to the Q-switching HV pulse applied to the Pockels Cell.

At last, we have developed a new type of picosecond laser which, in spite of its simple and quite conventional optical structure, is based on concepts that are novel and, possibly, far reaching. We believe that our device opens new perspective in the field of laser physics and technology.

\section{Acknowledgements}

This work has been supported by Gruppo Nazionale Elettronica Quantistica e Plasmi of Consiglio Nazionale delle Ricerche, Italy.

\section{References}

1. J. S. Liu, Optics Lett. 4, 372 (1979).

2. C. H. Brito Cruz, E. Palange and F. De Martini, Optics Comm. 39, 331 (1981); E. Palange, C. H. Brito Cruz, P. Di Lazzaro and F. De Martini, Appl. Phys. Lett. 41, 273 (1982).

3. J. E. Murray and D. J. Kuizenga, App. Phys. Lett. 37, 27 (1980); J. E. Murray, IEEE J. Quantum Electr. QE-17, 1713 (1981).

4. C. H. Brito Cruz, F. De Martini, H. L. Fragnito and E. Palange, Optics Comm. 40, 298 (1982).

5. C. H. Brito Cruz, F. De Martini and P. Mataloni, IEEE J. Quantum. Electr. QE-19, 573 (1983).

6. W. Koechner, Solid State Laser Engineering (Springer-Verlag, New York, Heidelberg, Berlin, 1976) Chapter 2, pp. 53-69.

7. KIGRE Q-100 phosphate glass. See KIGRE laser glass catalogue.

8. W. H. Lowdermilk, in: Laser Hardbook, ed. M. L. Stich (North Holland Publishing Company, 1979) pp. 361-420.

9. M. E. Mack, IEEE J. Quantum. Electr. QE-4, 1015 (1968).

10. C. V. Shank and E. P. Ippen, in: Ultrashort Light Pulses, ed. S. L. Shapiro (Springer Verlag, Berlin, 1977) pp. 83-122. 